\title{
Colonoscope incarceration in an inguinal hernia
}

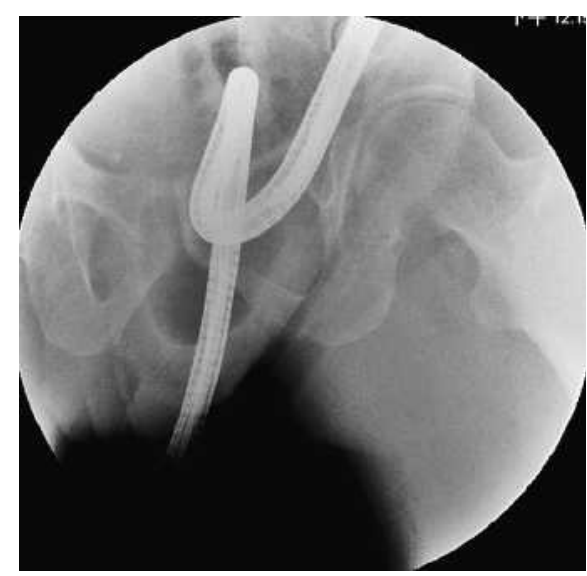

Figure 1 Under fluoroscopy, a U-shaped loop of the colonoscope was seen incarcerated in the left inguinal hernia.

A 73-year-old man who presented with symptoms of abdominal cramping and bloody stool passage underwent colonoscopy. After sedation, the procedure was carried out without difficulty, until at approximately $60 \mathrm{~cm}$, the colonoscope could not be advanced, although the lumen was clearly visible. Attempts to withdraw the scope failed and resulted in significant lower abdominal pain, as well as resistance to retrograde withdrawal. The patient was moved to the fluoroscopy room. Under fluoroscopy, a loop of colonoscope was seen in the left inguinal hernia (Figure 1). On examining the inguinal hernia sac, it was clear that the colonoscope was incarcerated within the hernia sac. The colonoscope could not be withdrawn directly while the loop of the scope within the hernia sac was in U-shape. But after forming an alpha loop in the hernia sac, by pushing and rotating the scope combined with careful withdrawal by gentle traction, the loop could be easily pushed into the abdominal cavity from the scrotum. Thus, we successfully reduced the incarcerated hernia.
In the English literature, only four other reports describe a colonoscope incarceration within a hernia sac [1-4]. Although it is rare, the colon is at risk within an incarcerated inguinal hernia. Careful history taking and physical examination, including the inguinal area, are important before undergoing colonoscopy. Although extraction of the colonoscope was difficult, the fluoroscope was an important tool in this case. We used the fluoroscope not only to estimate the width of the orifice of the hernia sac [2], but also to attempt to minimize the loop in the hernia sac. Although it could injure the fiber of the scope, we found that it was easier to minimize the loop by gently withdrawing the scope after forming an alpha loop. After the loop was minimized, the possibility of manual reduction could be estimated by comparing the size of loop with the width of the orifice of the hernia sac, under fluoroscopy. After reduction, it was possible to rotate the scope to release the alpha loop into the abdominal cavity, and withdraw it smoothly.

Endoscopy_UCTN_Code_CPL_1AJ_2AB

\section{S. Fan, M. S. Soon}

Department of Gastroenterology, Changhua Christian Medical Center, Taiwan.
References

${ }^{1}$ Ymamoto K, Kdakia SC. Incarceration of a colonoscope in an inguinal hernia. Gastrointest Endosc 1994; 40: 396-397

${ }^{2}$ Leisser A, Delpre G, Kadish U. Colonoscope incarceration: an avoidable event. Gastrointest Endosc 1990; 36: 637-638

${ }^{3}$ Leichtmann GA, Feingelrent H, Pomeranz IS, Novis BH. Colonoscopy in patients with large inguinal hernias. Gastrointest Endosc 1991; 37: 494

${ }^{4}$ Punnam SR, Ridout D. Incarcerated inguinal hernia. Gastrointest Endosc 2003; 58: 757 758

\section{Corresponding author}

\section{S. Fan, MD}

Department of Gastroenterology Changhua Christian Medical Center 135 Nanhsiao Street

Changhua 500

Taiwan

Fax: $\quad+886-47-228289$

Email:60909@cch.org.tw 